\title{
Management of Acute Demyelinating Attacks in the Pediatric Population: A Swiss Consensus Statement
}

\author{
Seline Hofer ${ }^{1}$, Florian Bauder ${ }^{2}{ }^{\infty}$, Andrea Capone Mori ${ }^{3}$, Andrew Chan ${ }^{4}$, Patricia Dill ${ }^{5}$, \\ Stéphanie Garcia-Tarodo ${ }^{6}$, Barbara Goeggel Simonetti ${ }^{4,7}$, Annette Hackenberg ${ }^{8}{ }^{\mathbb{D}}$, Judith Kalser 9 , \\ Oliver Maier ${ }^{10}$, Regula Schmid ${ }^{11}$, Susi Strozzi ${ }^{12}$, Sandra Bigi ${ }^{1,4,13, *}$ and on behalf of the "Medico Scientific \\ Advisory Board" of the Swiss Multiple Sclerosis Society ${ }^{\dagger}$
}

check for updates

Citation: Hofer, S.; Bauder, F.; Capone Mori, A.; Chan, A.; Dill, P.; Garcia-Tarodo, S.; Goeggel Simonetti, B.; Hackenberg, A.; Kalser, J.; Maier, O.; et al. Management of Acute Demyelinating Attacks in the Pediatric Population: A Swiss Consensus Statement. CTN 2021, 5, 17. https://doi.org/10.3390/ ctn5020017

Academic Editor: Claudio Bassetti

Received: 1 July 2021

Accepted: 31 August 2021

Published: 10 September 2021

Publisher's Note: MDPI stays neutral with regard to jurisdictional claims in published maps and institutional affiliations.

Copyright: (C) 2021 by the authors. Licensee MDPI, Basel, Switzerland. This article is an open access article distributed under the terms and conditions of the Creative Commons Attribution (CC BY) license (https:// creativecommons.org/licenses/by/ $4.0 /)$.
1 Division of Child Neurology, Department of Pediatrics, Children's Hospital Bern, Inselspital, University of Bern, 3010 Bern, Switzerland; seline.hofer.uwc@gmail.com

2 Children's Hospital, 6000 Lucerne, Switzerland; florian.bauder@luks.ch

3 Children's Hospital, 5001 Aarau, Switzerland; andrea.capone@ksa.ch

4 Department of Neurology, University Hospital, Inselspital, University of Bern, 3010 Bern, Switzerland; andrew.chan@insel.ch (A.C.); Barbara.GoeggelSimonetti@eoc.ch (B.G.S.)

5 Department of Pediatric Neurology, University Children's Hospital Basel, 4056 Basel, Switzerland; patricia.dill@ukbb.ch

6 University Children's Hospital, 1211 Geneva, Switzerland; Stephanie.Porri@hcuge.ch

7 Institute of Pediatrics of Southern Switzerland (IPSI EOC), 6500 Bellinzona, Switzerland

8 Department of Paediatric Neurology, University Children's Hospital, University of Zurich, 8032 Zurich, Switzerland; annette.hackenberg@kispi.uzh.ch

9 Pediatric Neurology and Neurorehabilitation Unit, Lausanne University Hospital, 1011 Lausanne, Switzerland; judith.kalser@chuv.ch

10 Children's Hospital, 9006 St. Gallen, Switzerland; oliver.maier@kispisg.ch

11 Children's Hospital, 8401 Winterthur, Switzerland; regula.schmid@ksw.ch

12 Department of Pediatrics, Cantonal Hospital, 7000 Chur, Switzerland; susi.strozzi@ksgr.ch

13 Institute of Social and Preventive Medicine, University of Bern, 3012 Bern, Switzerland

* Correspondence: sandra.bigi@insel.ch; Tel.: +41-31-63133-46

$\dagger$ Members are listed in acknowledgments.

Abstract: Background and methods: Acquired demyelinating syndromes (ADS) encompass distinct entities and occur in approximately 1/100,000 children. While the use of high dose intravenous corticosteroids is well-established, agreement on steroid taper and type of second line therapy is lacking. A comprehensive, unified and standardized treatment approach is crucial in the management of patients with rare diseases. Therefore, this study performed from July 2018 to June 2020 aimed at developing a national consensus on the management of ADS in the pediatric population using the Delphi approach. Consensus was defined as agreement in $>75 \%$. Designated Neuropediatricians with an expertise in the management of pediatric neuroinflammatory diseases in all university and cantonal hospitals of Switzerland were included. The response rate was 100\%. Results: High-dose i.v. methylprednisolone (20-30 mg/ $\mathrm{kg} /$ die for 5 days) is the first line treatment irrespective of the distinct entity of the ADS. An oral steroid taper is recommended in acute demyelinating encephalomyelitis (ADEM) and in neuromyelitis optica spectrum disorder (NMO-SD). However, in the latter more in the sense of bridging. The choice of second line treatment depends on the entity of ADS: in optic neuritis $(\mathrm{ON})$ and ADS due to relapsing remitting multiple sclerosis, first line treatment should be repeated, whereas plasma exchange is recommended in NMO-SD, ADEM and transverse myelitis. Conclusions: A national guideline allowing for a more unified approach in the management of pediatric ADS will enhance future research in this field, making data more comparable. The definition of inadequate treatment response to first line therapy remains a challenge and requires future research.

Keywords: demyelination; pediatric multiple sclerosis; corticosteroid treatment 


\section{Introduction}

Demyelinating diseases are distinct entities amongst inflammatory brain diseases (IBrainD) and encompass a broad spectrum of different disorders including optic neuritis (ON), transverse myelitis (TM), acute disseminated encephalomyelitis (ADEM), neuromyelitis optica spectrum disorders (NMO-SD) and pediatric onset relapsing remitting multiple sclerosis (RRMS) [1]. The first attack of all these entities is commonly described as acquired demyelinating syndrome (ADS). The pathophysiology is explained by a disruption of the blood-brain-barrier, leading to invasion of inflammatory cells and cytokines into the central nervous system (CNS) followed by an inflammatory reaction, as well as myelin edema and disruption [2-4].

Pediatric demyelinating diseases of the CNS are rare diseases. The incidence rates range between 0.07-2.9 per 100,000 children [5-7]. The epidemiological data for the Swiss pediatric population has yet to be established. The symptoms vary depending on the area of CNS inflammation [7-9]. The disease course can either be monophasic or relapsing [9]. Monophasic disease courses are often found to be of para/postinfectious or idiopathic origin, whereas a relapsing disease course represents a chronic inflammatory brain disease such as multiple sclerosis or neuromyelitis optica spectrum disorder [9].

Knowing about the potentially devastating impact of (relapsing) inflammatory attacks to the developing brain, a straightforward management of every single attack is required.

So far, there is agreement in the use of high-dose $(20-30 \mathrm{mg} / \mathrm{kg} /$ die $)$ i.v. methylprednisolone (MP) for 3-5 days in the case of an acute demyelinating attack [10-12]. Nevertheless, there is uncertainty if an oral prednisone taper following the i.v. MP treatment has any influence on outcome. To improve the care of children and adolescents with ADS it is important to identify and distinguish patients who will benefit from a prolonged steroid treatment from those where a taper is not necessary. Given the low incidences and hence difficulties in conducting randomized controlled trials, treatment approaches are primarily based on expert opinions. However, the recommendations differ between continents and even within countries.

An evaluation of the management for pediatric demyelinating diseases in the USA has shown that neurologists consider an oral prednisolone taper in 50-60\% of cases with ON, TM, ADEM or relapsing MS [10]. The IPMSSG (International Pediatric MS Study Group) recommends a prednisone taper for those MS patients not fully recovered after the i.v. MP course. On the other hand, there are pediatric MS centers not using a prednisone taper at all [11]. The definition of inadequate treatment response remains an additional challenge. A retrospective study on adult MS patients concluded that the use of an oral prednisone taper after i.v. MP did not improve disability or recovery in MS-related relapses [13]. To date, there are even less robust data on use of oral prednisone taper in the remaining demyelinating entities. Furthermore, the best choice of second-line treatment in case of treatment failure in this specific and vulnerable patient population needs to be identified.

In the field of rare diseases, a unified and structured treatment approach is crucial in clinical management, but also for research, where heterogeneity of individual data is a challenge. Therefore, this study aimed at a national consensus among the Swiss neuropediatricians in the management of pediatric ADS, focusing on first-line treatment, use of oral prednisone taper and choice of second-line treatment for every distinct demyelinating entity.

\section{Methods}

This study's cross-sectional design assessed the current management of acute demyelinating attacks nationwide in 2018 and used the Delphi approach to develop the consensus statement $[14,15]$. Consensus is defined as an agreement of over $75 \%$. Designated neuropediatricians with an expertise in the management of pediatric neuroinflammatory diseases in all university and cantonal hospitals of Switzerland were included $(n=11)$, covering all the neuropediatric centers of the Swiss national health care system. In one university hospital, the designated neuropediatrician changed between the first and the second survey due to retirement. A preliminary assessment revealed that ADS are not treated in 
private neuropediatric practices. Therefore, neuropediatricians in private practices were not further included in the consensus group. A first survey asked specific questions for each distinct ADS. These questions included number of patients under current care for an ADS, number of new ADS diagnosis per year (based on the experience over the past two years), dose and regimen of first-line treatment, use of oral prednisone taper (always, never, it depends), and choice of second-line therapy (repeat first-line therapy, plasma exchange, IVIG, others) in case of inadequate treatment response. Combining the results of the first survey with the current available literature, treatment recommendations were phrased for each demyelinating entity. These recommendations were again sent out to the Delphi group as a proposed consensus statement, seeking agreement on the individual recommendations (complete survey in Supplementary Table S1).

Continuous variables were summarized as mean (SD) or median (IQR) as appropriate, categorical variables are given as proportions (\%). The cumulative incidence rate per year was calculated using the population data from the Swiss Federal Office of Statistics [16].

\section{Results}

\subsection{First Survey}

The first survey was returned by all 11 participants, of whom $10(91 \%)$ provided answers to all questions. Overall, 71 patients were under ongoing care for an ADS in the Swiss neuropediatric institutions in 2018 (Table 1). A new diagnosis of ADS was made in 40 patients, most of them presenting with either optic neuritis (27.5\%) or ADEM (30\%). The calculated incidence rate of ADS in Switzerland is therefore approximately 2.77 per 100,000 children.

Table 1. Frequency of distinct acute demyelinating syndrome.

\begin{tabular}{ccc}
\hline ADS & $\begin{array}{c}\text { Patient Currently in } \\
\text { Participants Care n (\%) }\end{array}$ & New Diagnosis Per Year n (\%) \\
\hline Optic Neuritis & $18(25.4)$ & $11(27.5)$ \\
Transverse Myelitis & $8(11.3)$ & $6(15)$ \\
Acute demyelinating & $20(28.9)$ & $12(30)$ \\
encephalomyelitis & $22(30.3)$ & $9(22.5)$ \\
RRMS & $3(4.2)$ & $2(5)$ \\
NMO-SD & $71(100)$ & $40(100)$ \\
Total &
\end{tabular}

\subsubsection{First-Line Therapy}

More than $90 \%$ stated to use $20-30 \mathrm{mg} / \mathrm{kg} /$ die (max. $1000 \mathrm{mg} /$ die) i.v. MP for $3-5$ days. Of note, the possibility of not treating demyelinating attacks presenting with mild symptomatology in RRMS is also an option, but was not specifically investigated in our study.

\subsubsection{Taper Regimen}

The approach towards the use of an oral prednisone taper is handled heterogeneously and is presented in Table 2 . Almost $50 \%$ of the participants use an oral prednisone taper for all ADS. The remaining participants do not use an oral prednisone taper at all or take individual decisions depending on the clinical recovery after i.v. treatment. Excerpts of individual comments of the participants are presented at the bottom of Table 2. 
Table 2. The frequency of oral prednisone taper used per distinct acute demyelinating syndrome.

\begin{tabular}{cccc}
\hline ADS & Yes, Always n (\%) & It Depends * $\mathbf{n}(\mathbf{\%})$ & No $\mathbf{n}(\%)$ \\
\hline ON & $4(36.4)$ & $2(18.2)$ & $5(45.5)$ \\
TM & $5(50)$ & $3(30)$ & $2(20)$ \\
ADEM & $4(40)$ & $1(10)$ & $5(50)$ \\
RRMS & $4(40)$ & $2(20)$ & $4(40)$ \\
NMO-SD & $4(40)$ & $3(30)$ & $2(20)$ \\
\hline
\end{tabular}

${ }^{*} \mathrm{ON}$ : response to i.v. treatment // in recurrent ON. * TM: response to i.v. treatment // underlying disease // if not fully recovered, still residual symptoms but not as severe as to use ttm escalation. * ADEM: if not fully recovered. * RRMS: response to i.v. treatment. * NMO-SD: taper over 3 Months // Response to i.v. treatment.

\subsubsection{Second-Line Treatment}

Approximately $50 \%$ of the participants would repeat the first-line treatment for all ADS (Table 3). The use of IVIG as second-line treatment was limited to ON, TM and ADEM, whereas the use of plasma exchange in RRMS and NMO-SD mostly depended on the severity of residual clinical symptoms after first-line therapy.

Table 3. The use of second-line treatment.

\begin{tabular}{ccccc}
\hline ADS & $\begin{array}{c}\text { Repeat First Line } \\
\text { Treatment } \mathbf{~ ( \% )}\end{array}$ & $\begin{array}{c}\text { Plasma } \\
\text { Exchange } \mathbf{~ ( \% )}\end{array}$ & IVIG n (\%) & $\begin{array}{c}\text { Others, Please } \\
\text { Specify n (\%) }\end{array}$ \\
\hline ON & $5(50)$ & $1(10)$ & $3(30)$ & $1(10)$ \\
TM & $4(40)$ & $3(30)$ & $2(20)$ & $1(10)$ \\
ADEM & $5(50)$ & $1(10)$ & $2(20)$ & $1(10)$ \\
RRMS & $5(50)$ & $2(20)$ & - & $3(30)$ \\
NMO-SD & $4(40)$ & $3(30)$ & - & $3(30)$ \\
\hline
\end{tabular}

* ON + TM + ADEM: never needed. * RRMS: never necessary // Depending on severity of symptoms between Plasma exchange and repeat first line treatment. ${ }^{*}$ NMO-SD: repeat steroid or plasma exchange depending on severity of symptom.

\subsection{Second Survey}

The second survey yielded again a response rate of $100 \%$. The statement on first-line treatment of any ADS, i.e., $20-30 \mathrm{mg} / \mathrm{kg}$ i.v. MP ( $\max 1 \mathrm{~g} /$ die) for 5 days, reached an agreement of $100 \%$. In selected cases of restitutio ad integrum within 3 days, stopping the treatment early can be considered.

The statement on the use of oral prednisone taper reached an agreement of $100 \%$ in ON, TM, RRMS and NMO-SD and 91\% in ADEM. The group agrees on using an oral prednisone taper only in patients presenting with ADEM or NMO-SD. In cases of ON, TM and RRMS treating physicians should consider the use of immediate second-line treatment if patients are still symptomatic (EDSS $\geq 2$ after 2 weeks in case of RRMS) after first-line treatment.

The statement on the choice of second-line treatment was agreed on by $100 \%$ of the participants (Table 4). The achieved consensus was converted into a treatment algorithm (Figure 1).

Table 4. Choice of second-line treatment.

\begin{tabular}{cc}
\hline ADS & Statement \\
\hline ON + RRMS & $\begin{array}{r}\text { In case of insufficient response in an acute optic neuritis or RRMS, } \\
\text { repeat the first-line treatment with i.v. MP. If further treatment is } \\
\text { required, we suggest the use of plasma exchange. } \\
\text { In case of poor response to i.v. MP, we suggest using plasma } \\
\text { exchange in fulminant forms of ADEM and IVIG in the remainder. } \\
\text { In case of poor response to i.v. MP for TM or ADS in NMO-SD, we } \\
\text { suggest to use plasma exchange. }\end{array}$ \\
\hline
\end{tabular}




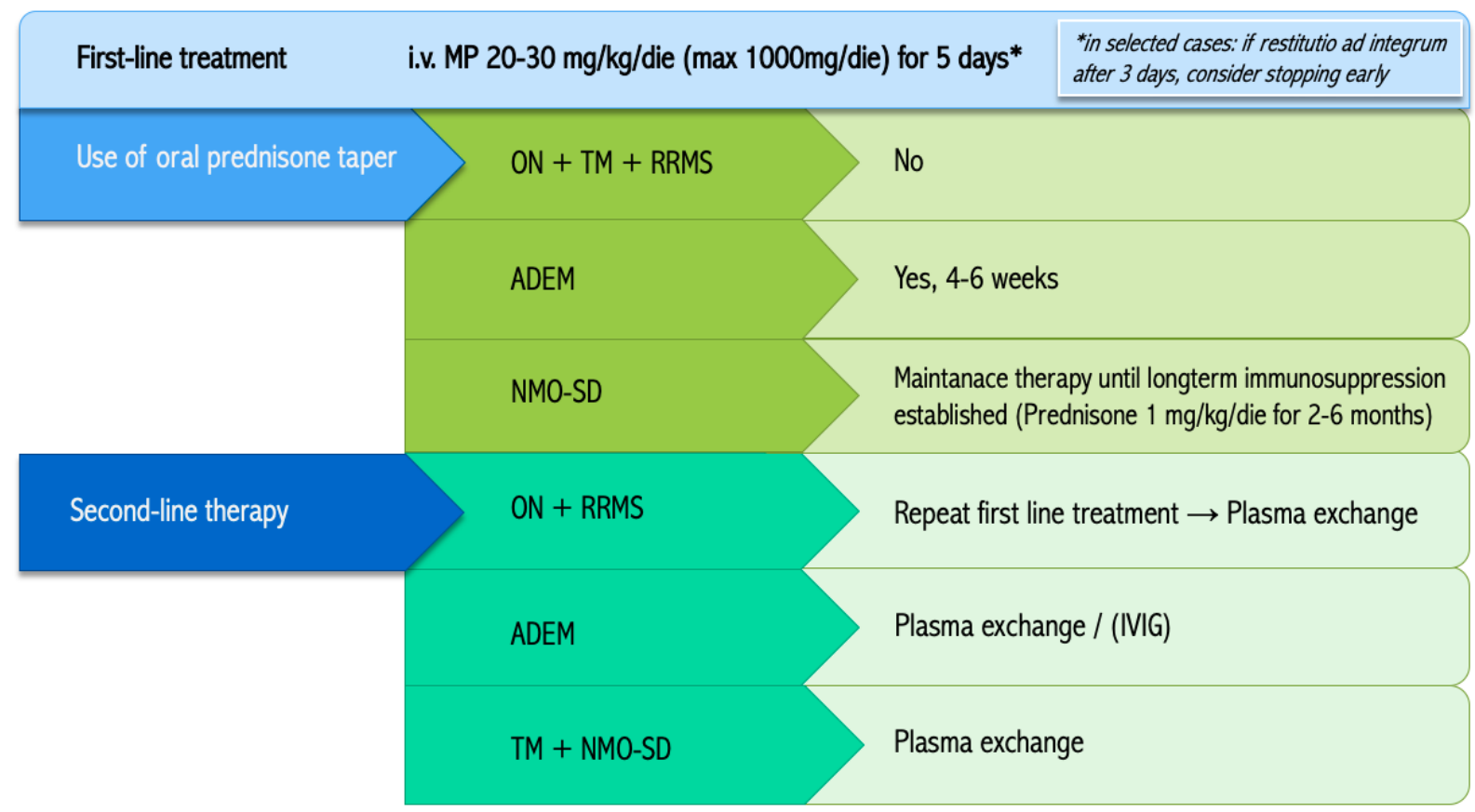

Figure 1. Treatment algorithm in pediatric acute demyelinating syndromes.

\section{Discussion}

The aim of this consensus was to develop a unified approach for the management of acute demyelinating attacks in the Swiss pediatric population. Using the Delphi method, the group agreed on a nationwide consensus on first and second-line therapies of pediatric ADS. The presented treatment algorithm will allow for a straightforward treatment of acute attacks in this particular area of rare diseases.

\subsection{Epidemiology: First Swiss Data on Incidence}

Although not the main aim of the project, the first survey included questions on the frequency of ADS in the individual centers. Assuming a steady rate of newly diagnosed ADS over the years, data from the survey was compared to the epidemiological data of the federal office of statistics for 2018 and revealed an incidence rate of 2.77 per 100,000 children. This is the first approximate calculation of an incidence of ADS in the Swiss pediatric population. Although it is in line with current numbers of other countries, it is important to mention that numbers derived from a simple survey and not out of a systematic search in the patient information systems $[5,6]$. Therefore, this data needs to be confirmed by more robust methodological techniques in the near future, such as the implementation of a national, population-based registry (www.swiss-ped-ibraind.ch). Active participation to the national registry is of utmost importance. The systematic and structured data collection allows better data quality, even beyond the core epidemiological questions. A greater number of patients enriches information on distinct disease entities and single events in the future and ultimately enhances care of this patient population. Furthermore, the registry can serve as a platform for future collaborative projects. As the conduction of a randomized controlled trial on this topic is not realistic, alternative approaches are required to obtain data on the effectiveness of treatment regimens such as pooling of data from different data sources or propensity score matching.

\subsection{The Oral Prednisone Taper: A Challenge}

The use of an oral prednisone taper remains controversial, most likely due to complete lack of evidence in the literature in pediatrics but also in adults. From an endocrinological point of view, a treatment with high dose i.v. MP for 3-5 days does not, as previously thought, require a prednisone taper and can be easily discontinued [17]. In case of $\mathrm{ON}$, the 
literature does not show a beneficial outcome for the use of an oral prednisone taper [18]. The same applies to TM (not associated with SLE or NMO-SD) and RRMS, where patients are not treated with an oral prednisone taper as supported by international literature $[10,19]$. In NMO-SD there is a clear indication for long-term immunosuppression as soon as the diagnosis has been confirmed. Depending on the chosen substance, there is a time lag between start of long-term immunosuppression and established immunosuppressive effect. Therefore, the continuation of oral prednisone in NMO-SD is more a bridging therapy of individual duration (i.e., until long-term immunosuppression is effective) than a short-term taper after high-dose i.v. MP [20,21].

The only entity that engaged discussion in the second survey was the use of a taper in ADEM. One participant addressed the lacking literature and the good clinical experience they had in not tapering their patients over the past years. However, some children with a more severe or stuttering course of ADEM and good response to i.v. MP could benefit from a slower weaning off from steroids. The fact that waxing and waning of symptoms in ADEM within the first three months after symptom onset are considered one acute episode might support the hypothesis of a beneficial taper. Therefore, the group suggested using the oral prednisone taper as described by Cole et al. (2019) and to carefully reassess the upcoming literature to adapt the statement to new findings [22-24].

Prolonged steroid treatment is known for its side effects such as weight gain, elevated blood pressure, hyperglycemia and sleep/mood disturbances $[25,26]$. Furthermore, steroid treatment is known to cause brain atrophy which is thought to be temporary. The impact of oral prednisone taper on brain atrophy is unknown $[27,28]$. Although the study did not investigate the influence of concerns about side effects of prolonged steroid treatment, any prolonged steroid treatment needs a careful risk-benefit evaluation.

\subsection{Applicability of the Consensus Statement: Benefits and Points of Caution}

Having a unified treatment algorithm in an area of rare diseases is clearly a benefit for both clinical care and research. A structured and straightforward treatment approach improves the management of acute demyelinating attacks by streamlining the therapy and avoiding unnecessary delays. Additionally, it enhances clinical information for research, making data more comparable and avoiding the large heterogeneity known to be a serious challenge for research in a field, where randomized controlled trials are difficult to conduct and projects are mainly based on observational studies. Furthermore, achieving a national consensus strengthens the network and collaboration amongst the experts in the field. This again will ultimately benefit the patients. Looking at the method, one could argue that the consensus group and the number of surveys (iterations) were too small for a regular Delphi process. This is partially true; however, we felt that the Delphi approach, i.e., reaching consensus among experts on a topic where evidence based data is lacking or not achievable, was the best way to reach our aim.

Although consensus of $>75 \%$ was achieved on all single statements, participants highlighted areas of particular caution. First, the treatment algorithm requires periodic evaluation and adaptation to novel findings in the scientific literature. This is of particular interest considering the rapid evolution in the field. Although findings on MRI such as lesion enhancement, lesion location and lesion burden can influence the individual treatment decision, it was not investigated separately in this study. In a future refinement of the consensus, the role of MRI on guiding therapeutic decisions should be included. This might be of particular interest in special situations such as ring-like enhancement or enhancement following steroid treatment. Second, the application of the algorithm requires caution to more recently described diseases, where pathophysiological processes might differ or clinical experience needs yet to be established. In this consensus, particularly MOG-Ab-positive disease was mentioned. MOG-Ab diseases can appear phenotypically, such as an ON, ADEM or AQP4-antibody negative NMO-SD, and disease courses are either monophasic or relapsing [29]. The initial level of antibody titer shows poor correlation with disease severity and/or risk for relapses. However, there is literature suggesting an 
association between clinical disease course and evolution of titers [30]. The resemblance of the disease course at least in some cases to NMO-SD and the fact of a detectable antibody prompt the treating physician towards a more aggressive treatment up front, often including IVIG and/or plasma exchange directly into the first-line treatment. Recently, the E. U. pediatric MOG-Consortium proposed an approach towards treatment of MOGAb-positive diseases [31]. Currently, the group suggests excluding MOG-positive patients from the algorithm until further evidence becomes available. Third, the survey showed that the driving force is the severity of residual clinical findings upon completion of i.v. MP for many decisions. The question arises of whether there is any additional benefit for recovery and particularly outcome in using a taper instead of nothing or a switch directly to second-line treatment. To tackle this question, the definition of treatment failure or inadequate treatment response to first-line treatment is of utmost importance. This definition remains a challenge, as it is not simply based on raw numbers, but also needs to account for the severity of the initial attack, the dynamics of recovery and the assumed underlying disease. Furthermore, we lack pediatric-specific outcome measures for this particular patient population. In this consensus, we proposed an EDSS cut-off of two in patients with a diagnosis of RRMS to decide on the use of second-line treatment. This is based on the experience that recovery in pediatric onset RRMS is usually excellent and overall EDSS scores are very low. Therefore, a persistent EDSS of $>2$ two weeks after i.v. MP can be considered a significant neurological deficit, justifying the evaluation of a second-line treatment.

The treatment algorithm was developed for the pediatric population, focusing on the particularities of pediatric treatments such as weight-adapted dose regimen. Yet, the general approach to demyelinating attacks is similar between the pediatric and adult population. Some differences do however apply, such as the higher threshold for the use of plasma exchange in the pediatric population due to invasiveness in smaller children (e.g., central catheter under general anesthesia).

\section{Conclusions}

The consensus provides a national treatment algorithm for first- and second-line treatment as well as the use of oral prednisone taper in pediatric ADS. In summary, the group agreed on: (1). High-dose i.v. methylprednisolone (20-30 mg/ $\mathrm{kg} / \mathrm{die}$ for 5 days) is the first line treatment irrespective of the distinct entity of the ADS. (2). An oral steroid taper is recommended in neuromyelitis optica spectrum disorder (NMO-SD) and acute demyelinating encephalomyelitis (ADEM). (3). The choice of second line treatment depends on the entity of ADS: in optic neuritis (ON) and ADS due to relapsing remitting multiple sclerosis, first line treatment should be repeated, whereas plasma exchange is recommended in NMO-SD, ADEM and transverse myelitis.

This national consensus strengthens clinical care and enhances clinical research in these rare diseases, making data more comparable in the future. Extending this consensus to a larger, i.e., European, setting could be of additional value in the management of pediatric demyelinating diseases. Furthermore, attempts are required to better define inadequate treatment responses to first-line treatment. Finally yet importantly, the consensus requires an update on a regular basis to include new clinical insights and implementation of advanced techniques such as MRI data.

Supplementary Materials: The following are available online at https://www.mdpi.com/article/ 10.3390/ctn5020017/s1, Table S1: Second survey.

Author Contributions: S.H. and S.B. researched literature, conceived the study and developed the protocol. F.B., A.C.M., A.C., P.D., S.G.-T., B.G.S., A.H., J.K., O.M., R.S., S.S. were involved in data collection, data analysis and critical scientific review of the manuscript. S.H. wrote the first draft of the manuscript. All authors reviewed and edited the manuscript and approved the final version of the manuscript.

Funding: There was no funding received for this study. 
Institutional Review Board Statement: Not applicable.

Informed Consent Statement: Not applicable.

Data Availability Statement: The data presented in this study are available in the article and Supplementary Material.

Acknowledgments: We thank the members of the Medico Scientific Advisory Board of the Swiss MS Society for their support: Achtnichts, Lutz, Cantonal Hospital Aarau, Neurology; Bansi, Jens, Rehabilitation centre Valens, Valens; Bigi, Sandra, Institute for Social and Preventive Medicine \& University Hospital Berne, Neurology; Biland-Thommen, Ursula, Neurophysio Biland/Kestenholz, Gelterkinden; Calabrese, Pasquale, University Basel, Psychology; Chan, Andrew, University Hospital Berne, Neurology; Czaplinski, Adam, Bellevue Medical Group \& Clinic for Neurology Hirslanden, Zürich; Derfuss, Tobias, University Hospital Basel, Neurology; Du Pasquier, Renaud, Centre hospitalier universitaire vaudois, Neurology; Engelhardt, Britta, University Berne, Theodor Kocher Institute; Gobbi, Claudio, Regional Hospital Lugano, Neurology; Gonzenbach, Roman, Rehabilitation centre Valens, Valens; Granziera, Cristina, University Hospital Basel, Neurology; Hoepner, Robert, University Hospital Berne, Neurology; Kamber, Nicole, University Hospital Berne, Neurology; Kamm, Christian, Cantonal Hospital Lucerne, Neurology; Kohler, Myrta, Ostschweizer Fachhochschule; Institut für Angewandte Pflegewissenschaft; Locatelli, Giuseppe, University Berne, Theodor Kocher Institute; Lutterotti, Andreas, Cellerys AG, Zürich; Luyckx, Kurt, Rehabilitation centre Valens, Valens; Mehling, Matthias, University Hospital Basel, Neurology; Müller, Stefanie, Cantonal Hospital St. Gallen, Neurology; Pot, Caroline, Centre hospitalier universitaire vaudois, Neurology; Puhan, Milo, University Zürich; Institute for Epidemiology, Biostatistics und Prevention; Rapold, Irene, MS patient representative; Salmen, Anke, University Hospital Berne, Neurology; Steinlin Egli, Regula, Physiotherapy Langmatten, Binningen; Théaudin, Marie, Centre hospitalier universitaire vaudois, Neurology; von Wyl, Viktor, University Zürich; Institute for Epidemiology, Biostatistics und Prevention; Zecca, Chiara, Regional Hospital Lugano, Neurology.

Conflicts of Interest: There are no financial or non-financial conflict. Andrew Chan has served on advisory boards for, and received funding for travel or speaker honoraria from Actelion-Janssen, Almirall, Bayer, Biogen, Celgene, Sanofi-Genzyme, Merck, Novartis, Roche and Teva, all for hospital research funds; and research support from Biogen, Genzyme and UCB. A. Chan is associate editor of the European Journal of Neurology and serves on the editorial board for Clinical and Translational Neuroscience and as topic editor for the Journal of International Medical Research. Sandra Bigi has served on advisory boards for, and received funding for travel or speaker honoraria from Novartis and Sanofi, all for hospital research funds; and received research support from Novartis and Roche.

\section{References}

1. Bar-Or, A.; Hintzen, R.Q.; Dale, R.C.; Rostasy, K.; Brück, W.; Chitnis, T. Immunopathophysiology of pediatric CNS inflammatory demyelinating diseases. Neurology 2016, 87, S12-S19. [CrossRef]

2. Metz, I.; Ms, S.D.W.; Popescu, B.F.G.; Frischer, J.; Parisi, J.E.; Guo, Y.; Lassmann, H.; Brück, W.; Lucchinetti, C.F. Pathologic heterogeneity persists in early active multiple sclerosis lesions. Ann. Neurol. 2014, 75, 728-738. [CrossRef]

3. Lucchinetti, C.F.; Popescu, B.F.; Bunyan, R.F.; Moll, N.M.; Roemer, S.F.; Lassmann, H.; Brück, W.; Parisi, J.E.; Scheithauer, B.W.; Giannini, C.; et al. Inflammatory Cortical Demyelination in Early Multiple Sclerosis. N. Engl. J. Med. 2011, 365, $2188-2197$. [CrossRef]

4. Young, N.P.; Weinshenker, B.G.; Parisi, J.E.; Scheithauer, B.; Giannini, C.; Roemer, S.F.; Thomsen, K.M.; Mandrekar, J.N.; Erickson, B.J.; Lucchinetti, C.F. Perivenous demyelination: Association with clinically defined acute disseminated encephalomyelitis and comparison with pathologically confirmed multiple sclerosis. Brain 2010, 133, 333-348. [CrossRef]

5. Banwell, B.; Kennedy, J.; Sadovnick, D.; Arnold, D.L.; Magalhaes, S.; Wambera, K.; Connolly, M.B.; Yager, J.; Mah, J.K.; Shah, N.; et al. Incidence of acquired demyelination of the CNS in Canadian children. Neurology 2009, 72, 232-239. [CrossRef] [PubMed]

6. Pohl, D.; Hennemuth, I.; Von Kries, R.; Hanefeld, F. Paediatric multiple sclerosis and acute disseminated encephalomyelitis in Germany: Results of a nationwide survey. Eur. J. Nucl. Med. Mol. Imaging 2007, 166, 405-412. [CrossRef]

7. Yeh, E.A.; Graves, J.S.; Benson, L.A.; Wassmer, E.; Waldman, A. Pediatric optic neuritis. Neurology 2016, 87, S53-S58. [CrossRef]

8. Absoud, M.; Greenberg, B.; Lim, M.; Lotze, T.; Thomas, T.; Deiva, K. Pediatric transverse myelitis. Neurology 2016, 87, S46-S52. [CrossRef] [PubMed]

9. Bigi, S.; Banwell, B. Pediatric Multiple Sclerosis. J. Child Neurol. 2012, 27, 1378-1383. [CrossRef] [PubMed]

10. Waldman, A.T.; Gorman, M.P.; Rensel, M.R.; Austin, T.E.; Hertz, D.P.; Kuntz, N.L.; Network of Pediatric Multiple Sclerosis Centers of Excellence of the National Multiple Sclerosis Society. Management of pediatric central nervous system demyelinating disorders: Consensus of United States neurologists. J. Child Neurol. 2011, 26, 675-682. [CrossRef] [PubMed] 
11. Huppke, P.; Gärtner, J. A Practical Guide to Pediatric Multiple Sclerosis. Neuropediatrics 2010, 41, 157-162. [CrossRef]

12. Dale, R.C.; Brilot, F.; Banwell, B. Pediatric central nervous system inflammatory demyelination: Acute disseminated encephalomyelitis, clinically isolated syndromes, neuromyelitis optica, and multiple sclerosis. Curr. Opin. Neurol. 2009, 22, 233-240. [CrossRef] [PubMed]

13. Perumal, J.S.; Caon, C.; Hreha, S.; Zabad, R.; Tselis, A.; Lisak, R.; Khan, O. Oral prednisone taper following intravenous steroids fails to improve disability or recovery from relapses in multiple sclerosis. Eur. J. Neurol. 2008, 15, 677-680. [CrossRef] [PubMed]

14. Okoli, C.; Pawlowski, S.D. The Delphi method as a research tool: An example, design considerations and applications. Inf. Manag.-Amster. 2004, 42, 15-29. [CrossRef]

15. Hasson, F.; Keeney, S.; McKenna, H. Research guidelines for the Delphi survey technique. J. Adv. Nurs. 2000, 32, 1008-1015. [CrossRef] [PubMed]

16. Bevölkerungsbestand per 31.12.2018. 2018. Available online: https://www.bfs.admin.ch/bfs/de/home/statistiken/ bevoelkerung/stand-entwicklung/bevoelkerung.assetdetail.14087554.html (accessed on 1 August 2020).

17. Levic, Z.; Micic, D.; Nikolić, J.; Stojisavljević, N.; Sokić, D.; Jankovic, S.; Kendereški, A.; Mavra, M. Short-term high dose steroid therapy does not affect the hypothalamic-pituitary-adrenal axis in relapsing multiple sclerosis patients. Clinical assessment by the insulin tolerance test. J. Endocrinol. Investig. 1996, 19, 30-34. [CrossRef]

18. Beck, R.W.; Cleary, P.A.; Anderson, M.M.; Keltner, J.L.; Shults, W.T.; Kaufman, D.I.; Buckley, E.G.; Corbett, J.J.; Kupersmith, M.J.; Miller, N.R.; et al. A Randomized, Controlled Trial of Corticosteroids in the Treatment of Acute Optic Neuritis. N. Engl. J. Med. 1992, 326, 581-588. [CrossRef]

19. Treatment of Acute Exacerbations of Multiple Sclerosis in Adults. 2019. Available online: https://www.uptodate.com (accessed on 19 April 2020).

20. Kimbrough, D.; Fujihara, K.; Jacob, A.; Lana-Peixoto, M.A.; Leite, M.I.; Levy, M.; Marignier, R.; Nakashima, I.; Palace, J.; de Seze, J.; et al. Treatment of neuromyelitis optica: Review and recommendations. Mult. Scler. Relat. Disord. 2012, 1, 180-187. [CrossRef]

21. Sellner, J.; Boggild, M.; Clanet, M.; Hintzen, R.Q.; Illes, Z.; Montalban, X.; Du Pasquier, R.; Polman, C.H.; Sorensen, P.S.; Hemmer, B. EFNS guidelines on diagnosis and management of neuromyelitis optica. Eur. J. Neurol. 2010, 17, 1019-1032. [CrossRef]

22. Pohl, D.; Alper, G.; Van Haren, K.; Kornberg, A.J.; Lucchinetti, C.F.; Tenembaum, S.; Belman, A.L. Acute disseminated encephalomyelitis: Updates on an inflammatory CNS syndrome. Neurology 2016, 87, S38-S45. [CrossRef]

23. Cole, J.; Evans, E.; Mwangi, M.; Mar, S. Acute Disseminated Encephalomyelitis in Children: An Updated Review Based on Current Diagnostic Criteria. Pediatr. Neurol. 2019, 100, 26-34. [CrossRef] [PubMed]

24. Anlar, B.; Guven, A.; Haspolat, S.; Yakut, A.; Serdaroglu, A.; Senbil, N.; Karaagaoglu, E.; Oguz, K.K.; Basaran, C.; Köse, G.; et al. Acute Disseminated Encephalomyelitis in Children: Outcome and Prognosis. Neuropediatrics 2003, 34, 194-199. [CrossRef] [PubMed]

25. Sloka, J.S.; Stefanelli, M. The mechanism of action of methylprednisolone in the treatment of multiple sclerosis. Mult. Scler. J. 2005, 11, 425-432. [CrossRef]

26. Rimsza, M.E. Complications of Corticosteroid Therapy. Arch. Pediatr. Adolesc. Med. 1978, 132, 806-810. [CrossRef] [PubMed]

27. Patil, C.G.; Lad, S.P.; Katznelson, L.; Laws, E.R., Jr. Brain atrophy and cognitive deficits in Cushing's disease. Neurosurg. Focus 2007, 23, E11. [CrossRef] [PubMed]

28. Zivadinov, R. Steroids and brain atrophy in multiple sclerosis. J. Neurol. Sci. 2005, 233, 73-81. [CrossRef] [PubMed]

29. Hacohen, Y.; Banwell, B. Treatment Approaches for MOG-Ab-Associated Demyelination in Children. Curr. Treat. Options Neurol. 2019, 21, 1-14. [CrossRef] [PubMed]

30. Armangue, T.; Capobianco, M.; de Chalus, A.; Laetitia, G.; Deiva, K.; Bruijstens, A.L.; Wendel, E.-M.; Lechner, C.; Bartels, F.; Finke, C.; et al. E.U. paediatric MOG consortium consensus: Part 3-Biomarkers of paediatric myelin oligodendrocyte glycoprotein antibody-associated disorders. Eur. J. Paediatr. Neurol. 2020, 29, 22-31. [CrossRef]

31. Bruijstens, A.L.; Wendel, E.-M.; Lechner, C.; Bartels, F.; Finke, C.; Breu, M.; Flet-Berliac, L.; de Chalus, A.; Adamsbaum, C.; Capobianco, M.; et al. E.U. paediatric MOG consortium consensus: Part 5-Treatment of paediatric myelin oligodendrocyte glycoprotein antibody-associated disorders. Eur. J. Paediatr. Neurol. 2020, 29, 41-53. [CrossRef] 